\title{
CALENDRIER POLLINIQUE ET RHINITE ALLERGIQUE À CAEN
}

\author{
E. BABIN, A. BELLOSO*, B. CHATIZEL, \\ C. JUNG, L. LEPELLETIER ${ }^{\star \star}$, E. EDY, A. BÉQUIGNON \\ SERVICE ORL ET DE CHIRURGIE CERVICO-FACIALE, CAEN, FRANCE \\ *DÉPARTEMENT D'ORL ET DE CHIRURGIE CERVICO-FACIALE, MANCHESTER ROYAL \\ INFIRMARY, ANGLETERRE. \\ ${ }^{* *}$ MÉDECIN ALLERGOLOGUE, CAEN, FRANCE
}

\begin{abstract}
OBJECTIF : Déterminer un calendrier pollinique local et enregistrer les manifestations cliniques allergiques observées dans la population durant cette même période

MÉTHODES : Cette étude prospective a été réalisée de la mi-février à la mi-septembre en 2001 et 2002 . Le calendrier pollinique de la région Caen/Basse-Normandie fut établi avec identification des pollens, leur date d'apparition, leur temps de présence et leur quantité. Parallèlement, des médecins locaux du Réseau National de Surveillance Aérobiologique (RNSA) enregistraient les manifestations cliniques allergiques (rhinites, conjonctivites, toux et asthme) dépistées dans leur consultation journalière.
\end{abstract}

RÉSULTATS : Sur Caen, la pollinisation débute avec les pollens de cupressaceae (thuyas) puis d' aulne (Alnus), puis se poursuit durant la première quinzaine d'avril avec les pollens de frêne (Fraxinus) et du bouleau (Betula), de chêne (Quercus) à la fin avril, des graminées (Poaceae) à la mi-juin et du châtaignier (Castenea) en juillet. Si le nombre total de taxons tous pollens confondus recueillis varie d'une année à l'autre, leur chronologie d'apparition est généralement la même.

Parallèlement en 2001, pendant la période de pollinisation des graminées, le pourcentage de patients consultant pour des symptômes allergiques était de $18,65 \%$ et de $9,1 \%$ pour une rhinite allergique. Par comparaison ces chiffres étaient respectivement égaux à $12,8 \%$ et $4,62 \%$ pendant toute la période d'observation.

En 2002, pendant la période de pollinisation des pollens de bouleaux, le pourcentage de patients consultant pour des symptômes allergiques était de $15,5 \%$ et de $7,2 \%$ pour une rhinite allergique. Par comparaison ces chiffres étaient respectivement égaux à $10,62 \%$ et $3,48 \%$ pendant toute la période d'observation.

COMMENTAIRES : Tous ces résultats sont en accord avec les différentes études réalisées entre 1978 et 1999 et démontrent une relation entre les comptes polliniques et l'incidence des manifestations cliniques allergiques.

CONCLUSION : Ce travail a permis d'établir une corélation entre le calendrier pollinique et les manifestations cliniques allergiques enregistrées sur la région caennaise pendant la période d'observation et d'identifier des périodes à risques allergiques.

MOTS CLES : Calendrier pollinique, allergie, rhinite allergique

\section{SUMMARY}

OBJECTIVE:Toestablish a local pollinic calendar of Caen (France), contrasting those results with allergic symptoms observed in the local population

METHODS: Prospective cohort study during the months of February to September in 2001 and 2002. A local pollinic calendar of Caen (France) was establish identifying the nature and concentration of the pollens, date of identification and length of detection. Simultaneously, allergic clinical manifestations in the local population (rhinitis, conjunctivitis, asthma) were documented by specialists of the local Réseau National de Surveillance Aérobiologique (RNSA).

RESULTS: In Caen, pollination started in the beginning of March with pollens of conifers (Thuja) and alnus (alder), following by fraxinus (ash) and betula (birch) in the first half of April. Quercus (oak) was detected in the end of April. Pollens of graminea (grasses) were detected in the middle of June and castenea (chestnut) in July. The concentration of pollens was variable in both years, but the dates of detection were similar.

In $2001,9.1 \%$ (compared with a yearly mean of $4.62 \%$ ) was the highest percentage of clinical consultations for allergic rhinitis and corresponded to the period of pollination of the gramineas. During this period the percentages of consultation for other allergic symptoms was $18.65 \%$, comparing with a mean of $12.8 \%$ during the year.

In $2002,7.2 \%$ (compared with a yearly mean of $3.48 \%$ ) was the highest percentage of clinical consultations for allergic rhinitis and corresponded to the period of pollination of the betula. During this period the percentages of consultation for other allergic symptoms was $15.5 \%$, comparing with a mean of $10.62 \%$ during the year.

DISCUSSION: Those findings correspond with previous published studies from 1978 to 1999 , demonstrating a relation between concentration of pollens and incidence of allergic symptoms.

CONCLUSIONS: The study establishes a correlation between the pollinic calendar and the allergic manifestation detected in local population. Prediction, by a yearly-published pollination calendar, of the precise periods of high concentration of specific pollens can alert the risk population to adopt measures of avoidance or contemplate prophylactic treatment. KEY WORDS: Pollination calendar, allergy, allergic rhinitis. 


\section{INTRODUCTION}

La Rhinite Allergique est une inflammation de la muqueuse nasale due à une réaction immunologique spécifique Immunoglobuline $\mathrm{E}$ (IgE) dépendante qui survient quand la muqueuse est exposée à certaines particules aéroportées: les pneumallergènes. Elle touche 10 à $40 \%$ de la population dans le monde. D'autres symptômes y sont associés, comme l'asthme, la conjonctivite, la toux, les prurits, etc. $[1,2]$. La part de la rhinite allergique pollinique est d'environ $50 \%$ et touche de 8 à $20 \%$ de la population générale [3]. Ces pollens ne sévissent pas de façon uniforme toute l'année mais par périodes, la plus gênante est due aux graminées de mai à début juillet [4]. En raison de la variabilité de la pollinisation, le Réseau National de Surveillance Aérobiologique (RNSA)* a décidé d'installer une quarantaine de capteurs en France pour que chaque région ait son propre calendrier pollinique.

Un capteur a été installé à Caen depuis 1999. Notre travail se proposait d'établir un calendrier pollinique local et de regrouper les symptômes allergiques observés avec l'aide de médecins de la région Caennaise.

L'objectif était de rechercher une corrélation entre le calendrier pollinique et les manifestations allergiques de la population caennaise.

"Le réseau national de surveillance aérobiologique (R.N.S.A) est une association (loi de 1901), crée en 1996 pour poursuivre les travaux réalisés depuis 1985 par le laboratoire d'aérobiologie de l'Institut Pasteur à Paris. Ce réseau a pour objet principal l'étude du contenu de l'air en particules biologiques pouvant avoir une incidence sur le risque allergique pour la population ; c'est à dire l'étude du contenu de l'air en pollens et en moisissures ainsi que du recueil des données cliniques associées.

Le R.N.S.A fonctionne grâce à un conseil scientifique composé de douze membres reconnus pour leur compétence dans le domaine couvert par l'association. Ils appartiennent à différents organismes ou institutions. Parmi eux figurent des analystes, des métrologistes, des médecins, et des ingénieurs. Les membres du conseil scientifique sont nommés par des organismes tel que La Direction Générale de la Santé, le Réseau National de Santé Publique, le Comité Supérieur d'Hygiène Publique, ainsi que des spécialistes en allergologies, en palynologie et en analyses biologiques. Ils sont renouvelés par moitié tous les deux ans.

Le R.N.S.A dispose d'un budget de 500000 euros provenant pour la moitié de subventions publiques et pour le reste de ressources privées (Laboratoires pharmaceutiques).

\section{MATÉRIEL ET MÉTHODES Le calendrier pollinique \\ - Lieu d'etude}

Caen est la capitale industrielle de Basse-Normandie. La préfecture du Calvados, située à $14 \mathrm{~km}$ de la mer est aujourd'hui une cité universitaire qui acceuille plus de 30000 étudiants sur 3 campus et compte 117000 habitants. Encadrée à l'ouest par le massif armoricain et à l'est par le bassin parisien, les reliefs différencés de la Basse-Normandie sont à l'origine des constrastes climatiques régionaux. Contrairement aux idées toutefaites donnant à la Normandie l'image d'une contrée verdoyante et largement arrosée, il n'existe pas à proprement parler de climat bas-normand mais plusieurs hydro-climats, les précipitations constituent le facteur prépondérant dans ces contrastes. L'exposition à la façade atlantique s'accompagne d'entrée d'air maritime poussé par les vents dominants d'ouest et ouest-sud-ouest. L'est de la région (dont la plaine de Caen) bénéficie du phénoméne d'abri occasioné par les hauteurs du Cotentin. L'agglomération caennaise est centrée sur une plaine à vocation agricole. Cette plaine est bordée à l'est par le Pays d'Auge et les marais de la Dives, à l'ouest par le bocage et la forêt de Cerisy, au nord par les côtes de la Manche et au sud par la forêt de Grimbost et plus loin, les reliefs de la Suisse Normande. Plus localement, l'agglomération caennaise dispose de jardins et d'espaces vert importants qui sont des lieux de promenade.

\section{- Type de végétation et nature des pollens}

La plaine de Caen, constituée de grandes cuitures est bordée à l'ouest comme à l'est de bocages à Ormes et Chênes. Les pollens susceptibles d'être capturés à Caen sont les suivants: pour les arbres, ce sont les pollens de tuyas, noisetiers, aulne, peuplier, orme, saule, frêne, charme, bouleau, hétre, chêne, pin, tilleul et chataigner. Pour les herbacées, ce sont l'oseille, le plantin, le pariétaire, les orties, les chenopodes et les armoises.

\section{- Le capteur.}

L'appareil utilisé pour le comptage des pollens repose sur une méthode volumétrique par aspiration : la trappe de Hirst, conçue en 1952. C'est l'appareil le plus répandu en Europe. Le principe de la méthode volumétrique repose sur l'aspiration de volumes d'airs connus, ce qui permet de rapporter le nombre de grains recueillis à l'unité de volume d'air [5].

Le capteur comprend 3 parties : (Figure 1)

La partie supérieure mobile est constituée d'un corps central muni d'un orifice à travers lequel est aspiré l'air. Cet orifice est protégé de la pluie par une feuille de métal faisant visière. Une petite vis permet de régler le débit d'aspiration. L'orifice d'entrée d'air est maintenu face au vent au moyen d'une girouette. Le sommet du capteur central est fermé par un disque métallique muni d'une peignée, sous lequel est fixé un tambour. Ce dernier est constitué d'un cylindre associé à un système d'horloger tournant à une vitesse de $2 \mathrm{~mm}$ par heure avec une autonomie de 7 jours. Sur ce tambour, est fixé chaque semaine, un film de Cellophane enduit d'une substance adhésive qui piège les particules aéroportées. La pompe aspirante est située dans la partie inférieure de l'appareil. Elle est réglée pour aspirer un volume de 10 litres d'air à la minute, ce qui correspond à la respiration moyenne humaine. 

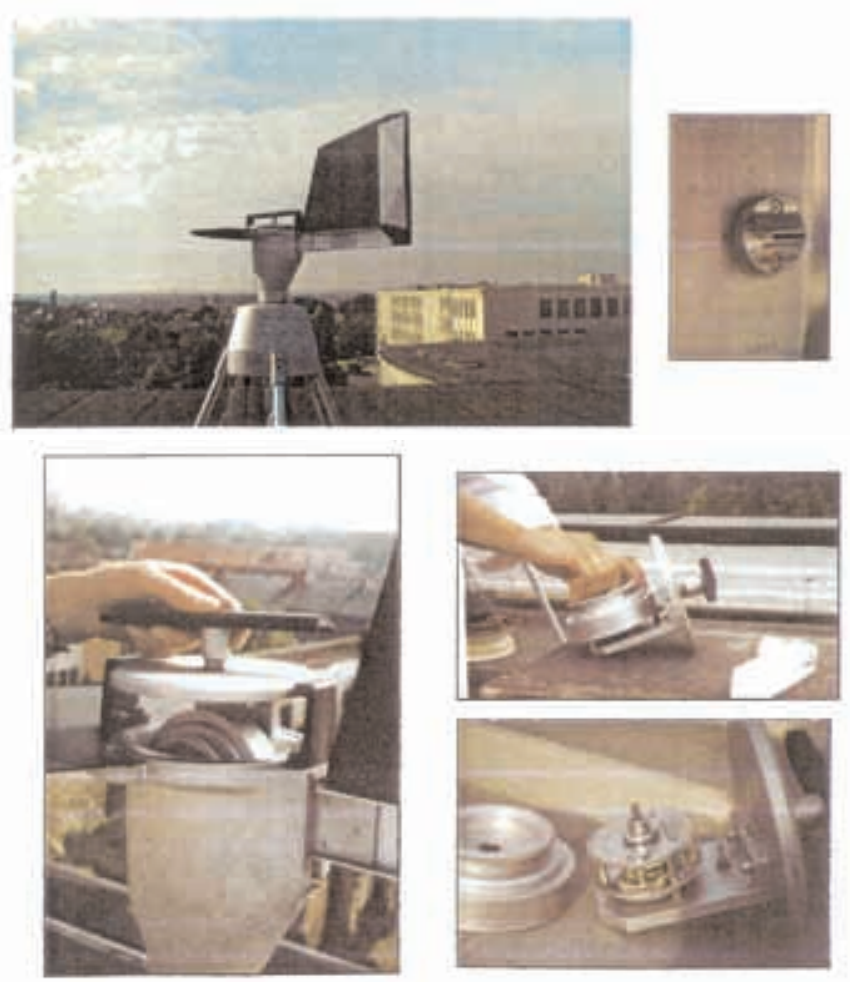

Fig. 1 : Capteur de pollens - Site de Caen

\section{- L'emplacement du capteur}

L'appareil de capture des pollens est situé au centre ville de Caen sur le toit de l'Université. La zone concernée par ce capteur est un cercle centré sur Caen et de rayon de $50 \mathrm{~km}$. Le choix de l'emplacement du capteur est d'une grande importance, c'est lui qui conditionne la récolte. Si l'appareil est très haut placé, les grains de pollen d'arbres prédominent. Au contraire, en situation trop basse, les grains de pollen d'herbacées sont essentiellement piégés. Il est préférable d'éviter la proximité d'un parc, il est également souhaitable de considérer la topologie du lieu. II faut éviter la proximité d'une source de souillure (orifice de cheminée) qui gênerait la lecture de la lame. L'université de Caen correspondait bien à ces critères, il a donc été décidé d'installer le capteur sur l'une des terrasses du campus à 17 mètres de hauteur par rapport au sol, lieu qui en outre se trouve à côté du laboratoire.

\section{- La méthode de recueil}

Les tambours sont relevés chaque semaine, à heure fixe. La bande adhésive est alors découpée en 7 segments (chaque segment correspondant à 1 jour) pour être analysée sous microscope optique. Une méthode d'échantillonnage est effectuée pour éviter la lecture de la totalité de la bande. En France, les comptes polliniques sont donnés par tranche de deux heures. Pour faciliter la lecture au microscope, un réactif colorant est utilisé pour distinguer les pollens (qui deviennent roses) des multiples autres particules aéroportées. Puis, une fiche de résultats journaliers est établie pour les 5 à 10 principaux taxons. Une moyenne est calculée chaque semaine du début mars à la fin de septembre. L'analyste responsable du capteur transmet cette fiche de résultats au R.N.S.A qui publie sur son site internet, aux médecins du réseau, à la presse et aux organismes de santé publique un bulletin allergo-pollinique avec un risque allergique (RA) variant de 1 à 5 ( 0 = risque nul, $1=$ risque très faible, $2=$ risque faible, $3=$ Risque moyen, 4 = risque élevé et 5 = risque très élevé). Ce risque est élaboré chaque semaine en incorporant, les données polliniques (quantité absolue de pollens mais aussi période de décroissance de stagnation ou de croissance), les données climatiques, et les prévisions météorologiques. Des commentaires divers complètent ces informations numériques : prévisions pour la semaine à venir, gravité des symptômes, nécessité de renforcer la vigilance voire le traitement ou au contraire diminuer ou arrêter les thérapeutiques préventives.

\section{Le calendrier des symptômes et de la rhinite aller-} gique.

Sept médecins sentinelles de la région Caennaise (trois médecins généralistes, un ORL, un allergologue et deux pédiatres) ont récolté des informations sur les symptômes évocateurs de pollinoses parmi leur clientèle. Ils ont ainsi de mi-février à mi-septembre, comptabilisé tous les symptômes suivants évocateurs d'allergie : rhinites, conjonctivites, asthme et toux. Aucune consigne particulière n'avait été donnée pour classer les symptômes observés comme étant d'origine pollinique. Chaque médecin utilisait la méthode qu'il désirait. Aucun examen complémentaire n'était demandé pour justifier son choix. Pour faciliter le travail des médecins, une fiche de recueil de données était fournie permettant de retranscrire leurs observations ainsi que le nombre total de patients vus.

Nous avons pu ainsi calculer le pourcentage de patients vus et le pourcentage de rhinites, de toux, d'asthme et de conjonctivite, observé parmi les consultants.

\section{RESULTATS}

En 2001, notre étude s'est déroulée sur 22 semaines et a comptabilisé 10710 patients (485 par semaine). En 2002, elle a retenu 11943 patients sur 29 semaines (411 patients par semaine).

L'expression des résultats était traduite sous forme de quatre graphiques :

1) Evolution des rhinites allergiques comparativement au calendrier pollinique au cours de l'année 2001. (Figure 2) 2) Evolution des symptômes allergiques comparativement au calendrier pollinique au cours de l'année 2001(Figure3)

3) Evolution des rhinites allergiques comparativement au calendrier pollinique au cours de l'année 2002. (Figure 4) 4) Evolution des symptômes allergiques comparativement au calendrier pollinique au cours de l'année 2002(Figure5) 


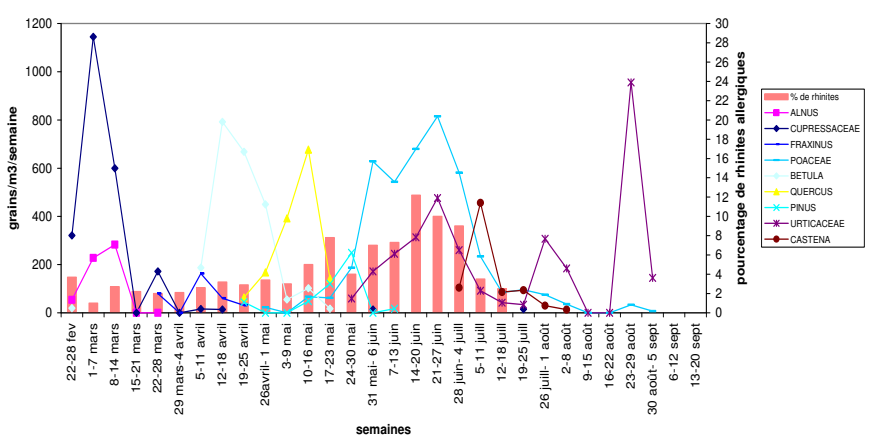

Fig. 2 : Evolution des rhinites allergiques comparativement au calendrier pollinique au cours de l'année 2001

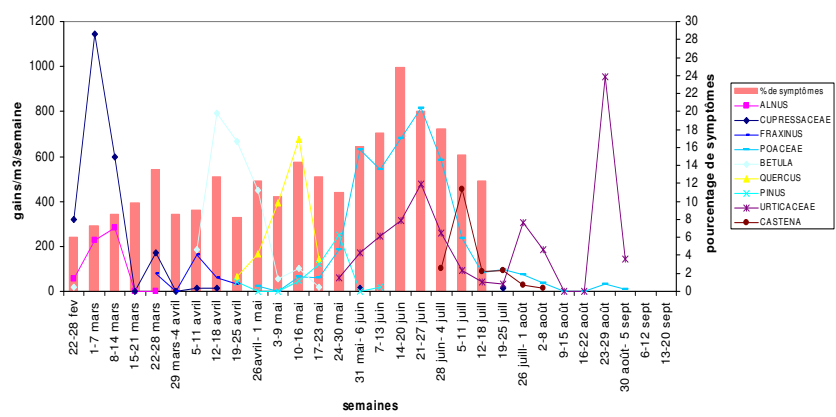

Fig. 3 : Evolution des symptômes allergiques comparativement au calendrier pollinique au cours de l'année 2001

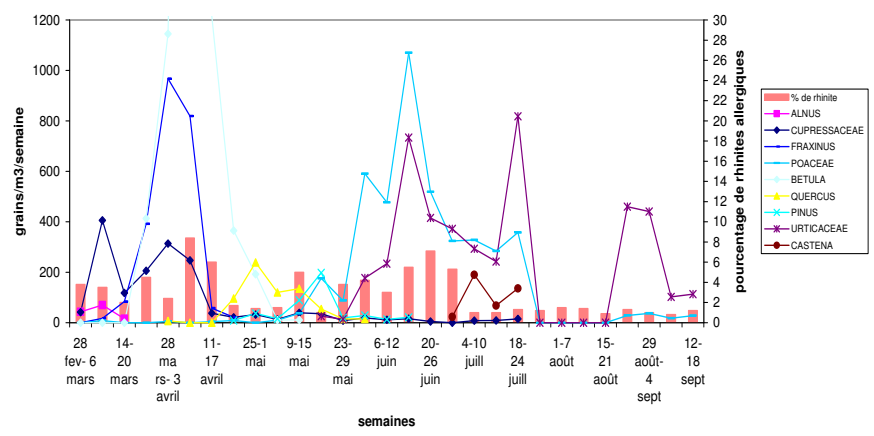

Fig. 4 : Evolution des rhinites allergiques comparativement au calendrier pollinique au cours de l'année 2002

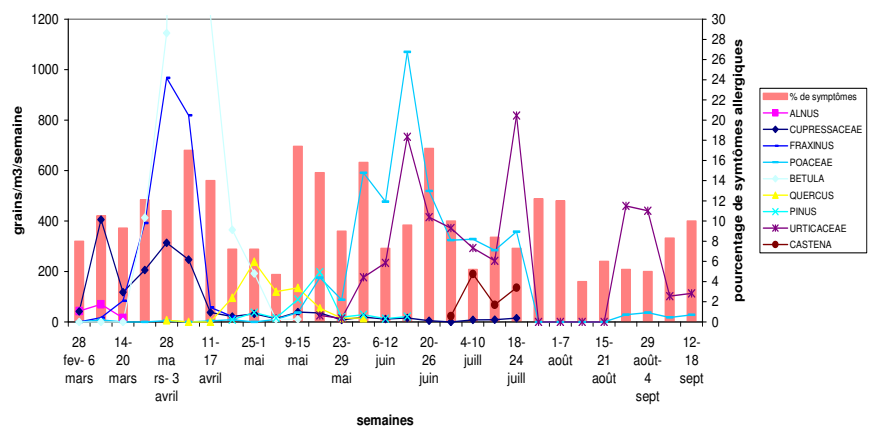

Fig. 5 : Evolution des symptômes allergiques comparativement au calendrier pollinique au cours de l'année 2002

\section{COMMENTAIRES}

\section{Comparaison intercapteurs $[6,7,8]$}

Caen est une ville où il y a peu de pollens (probablement à cause de la proximité de la mer à $14 \mathrm{~km}$ ). En 2001 elle était en 35ème position sur 46 villes évaluées avec 17000 grains/m3 /an, loin derrière Toulon ( 74000 grains/m3/an), mais devant Brest (7200 grains/m3). En 2002, elle se classait 33ème sur 45 villes avec 26000 grains/m3/an, derrière les villes de Bordeaux, Dijon, Marseille, Montluçon et Toulouse dont les capteurs ont absorbé plus de 50000 grains $/ \mathrm{m} 3$ lan et devant les villes de Brest, Briançon, Gap, Perpignan où le nombre de grains de pollens dépassait à peine 10000 grains $/ \mathrm{m} 3 / \mathrm{an}$.

Caen et les villes du nord de la Loire sont peuplées des mêmes familles de pollens : aulne, frêne, bouleau, chêne, graminées, orties, châtaignier, platane qui " pollinisent " à la même période c'est-à-dire durant la première quinzaine d'avril pour le frêne et le bouleau, fin avril pour le chêne, mi-juin pour les graminées et juillet pour les châtaigniers. En Basse-Normandie, les mêmes pollens arrivent au même moment à Caen et à La Ferté-Macé [9], située à $80 \mathrm{~km}$ au sud et longtemps la ville de référence. Cependant, la quantité globale récoltée est plus importante à la Ferté-Macé avec 34000 grains/m3/an contre 17000 grains/m3/an à Caen en 2001 et respectivement 38000 grains/m3/an contre 26000 grains/m3/an en 2002. Mais c'est surtout la quantité de pollens journellement recueillie (bruit de fond) qui est plus importante à la FertéMacé. Les pics polliniques sont assez équivalents en quantité. Ainsi pour l'année 2002, le pic de pollens pour le bouleau à Caen était égal à 1800 grains $/ \mathrm{m} 3 /$ j contre 1200 grains $/ \mathrm{m} 3 / \mathrm{j}$ à la Ferté-Macé et celui des pollens des graminées égale à 400 grains $/ \mathrm{m} 3 / \mathrm{j}$ à Caen contre 650 grains/m3/j à la Ferté-Macé).

Au sud de la Loire, les pollens diffèrent de manière assez sensible de ceux retrouvés dans la région caennaise [9]. Beaucoup de pollens de cyprès sont inexistants en février dans la capitale de Guillaume le Conquérant. Les pollens de cupressacées recueillis sont des pollens de thuyas. A Caen, les urticacées recueillis sont essentiellement de l'ortie contre les pollens de pariétaire au sud de la Loire. Les pollens d'ambroisie, absents au nord sont retrouvés en août dans le couloir Rhodanien. Enfin, la pollinisation au sud de la Loire retrouve des pollens de chêne, de graminées, mais peu de pollens de bouleau. La période de pollinisation est la même à 15 jours près.

Au total, Caen est une ville faiblement pollinisée. La nature et la date de pollinisation sont assez semblables à celle des autres villes du nord de la Loire. Par opposition, le sud de la Loire est pourvu de pollens très allergisants en particulier les pollens de cyprès, de pariétaire et d'ambroisie qui n'existent pas à Caen.

Une comparaison avec le bulletin pollinique de la FertéMacé, à $80 \mathrm{~km}$ au sud de Caen, permet d'établir un rôle joué par la proximité de la mer, avec une diminution de la 
quantité journalière de pollens recueillis (bruit de fond). Par contre, les pics polliniques sont en quantité, globalement les mêmes. Le calendrier pollinique de Caen apparait caractérisé par des pics biens marqués avec des quantités faibles de pollens entre les pics.

\section{Comparaison des 2 années polliniques à Caen.}

En 2002, le nombre total de taxons, tous pollens confondus recueillis sur les lames était plus important qu'en 2001 : $26000 \mathrm{gr} / \mathrm{m} 3 / \mathrm{an}$ contre $17000 \mathrm{gr} / \mathrm{m} 3 / \mathrm{an}$ en 2001. La chronologie d'apparition des différents taxons était généralement la même pour ces deux années, avec jamais plus d'une semaine d'écart. La saison de pollinisation débutait avec les pollens de cupressaceae (thuyas) puis d'alnus (aulne), de frêne, de bouleau, de chêne, de pin, de graminées, d'urticaceae (orties) et de châtaignier. En revanche, le pic pollinique, la quantité de pollen dans l'air, la durée de pollinisation diffèraient légèrement pour chaque taxon. Ainsi, le pic pollinique pour le Chêne était décalé de 2 semaines. La quantité des grains récoltés pouvait varier du simple au septuple et particulièrement pour les pollens d'arbres : la quantité de pollen de Bouleau et de Frêne était respectivement 7 et 6 fois plus importante en 2002 ; la quantité de pollens de thuyas, de chêne et d'aulne était 3 fois supérieur en 2001 par rapport à 2002. Enfin, une variation moindre est observée avec la durée de pollinisation, sensiblement la même à 1 semaine près sauf pour les pollens de graminées ( 5 semaines d'écart).

\section{Analyse des deux années avec «symptômes aller- giques"}

En 2001, de la mi-février à la mi-septembre, les médecins du réseau de surveillance ont observé $12,8 \%$ de consultants porteurs de symptômes allergiques et $4,62 \%$ souffrant de rhinite allergique. Par comparaison, durant la même période en 2002 , les chiffres étaient respectivement de $10,62 \%$ et $3,48 \%$.

Au cours de l'année 2001, Le pourcentage maximum de patients consultant pour des symptômes allergiques était de $18,65 \%$ (période du 31 mai au 12 juillet) avec un point culminant à $24,90 \%$ pendant la semaine du 14 au 20 juin. De même, le pourcentage maximum de patients consultant pour une rhinite allergique était de 9,1\% (période du 31 mai au 5 juillet) avec un point culminant à $12,2 \%$ pendant la semaine du 14 au 20 juin. Cette période correspondait exactement à la période de pollinisation des pollens de graminées à plus de 300 grains $/ \mathrm{m} 3 / \mathrm{semai}-$ ne.

En 2002, le pourcentage maximum de patients consultant pour des symptômes allergiques était de $15,5 \%$ (période du 3 avril au 17 avril) avec un point culminant à 17\% la semaine du 3 au 9 avril, et le pourcentage maximum de patients consultant pour une rhinite allergique était de $7,2 \%$ (période du 3 au 17 avril) avec un point culminant à
$8,4 \%$ pendant la semaine du 3 au 9 avril. Cette période correspondait à la période de pollinisation des pollens de bouleaux

Il est intéressant de noter que les périodes de pollinisation responsables de manifestations cliniques majeures sont corrélées à la pollinisation par les graminées en 2001 et à celle du bouleau en 2002.

\section{Détermination de doses seuils.}

La comparaison des deux calendriers (pollinisation et manifestations cliniques) a permis de déterminer des doses seuils responsables de symptômes allergiques.

En 2001, deux pollens apparaissaient particulièrement allergisants : le pollen de chêne avec des manifestations cliniques à partir d'un seuil de 400 grains $/ \mathrm{m} 3 / \mathrm{semaine}$ ( 60 grains $/ \mathrm{m} 3 /$ jour) et le pollen des graminées avec une symptomatologie allergique dès que le seuil dépassait 300 grains $/ \mathrm{m} 3 /$ semaine ( 40 grains $/ \mathrm{m} 3 /$ jour).

En 2002, deux pollens étaient très allergisants : le pollen de bouleau, dont le seuil est difficile à préciser exactement car le pic était étroit et la concentration de pollen dans l'air variait très vite d'une semaine à l'autre. Mais si à 400 grains $/ \mathrm{m} 3 / \mathrm{semaine}$, les manifestations cliniques étaient faibles, le taux de 1100 grains $/ \mathrm{m} 3 / \mathrm{semaine}$ était corrélé à une augmentation de la symptomatologie allergique (les taux symptomatiques correspondent à des seuils compris entre 60 et 160 grains $/ \mathrm{m} 3 /$ jour). De même, les pollens de graminées étaient responsables de manifestations cliniques plus importantes quand le seuil dépassait 400 grains $/ \mathrm{m} 3 /$ semaine (60 grains/m3/jour). $\mathrm{Par}$ comparaison, le pollen de chêne n'a pas entraîné de manifestations allergiques en 2002 ; mais ce seuil est resté en dessous de celui enregistré l'année précédente. Tous ces résultats sont en accord avec les différentes études réalisées entre 1978 et 1999 qui démontrent une relation entre les comptes polliniques et l'incidence de manifestations cliniques [10]. Ce phénomène est particulièrement caractéristique pour les pollens d'ambroisie, de graminées, de pariétaire et de bouleau. Dans notre étude la relation dose/seuil et manifestations cliniques est particulièrement évidente pour les pollens de graminées (en 2001 et 2002), de bouleau (en 2002), et de chêne (en 2001).

Ce calcul de dose/seuils de pollinisation responsable de manifestations cliniques est pour les pollens de bouleau compris entre 60 et 80 grains $/ \mathrm{m} 3 /$ jour l'apparition de symptômes allergiques et de 35 à 205 grains $/ \mathrm{m} 3 /$ jour pour la survenue de rhinites allergiques. Pour les pollens de graminées, le seuil d'apparition d'une symptomatologie clinique allergique est compris entre 15 à 20 grains/m3/jour. Un seuil compris entre 25 et 81 grains $/ \mathrm{m} 3 /$ jour est corrélé avec une augmentation franche des manifestations cliniques. 


\section{CONCLUSION}

L'intérêt de ce travail était d'établir une corélation entre le calendrier pollinique de la ville de Caen (identification des pollens et de leur seuil allergisant) et les manifestations cliniques allergiques enregistrées sur la région caennaise pendant la période d'observation. Cette étude a ainsi permis d'identifier des périodes à risques allergiques.

Cependant, ces résultats doivent être interprétés avec beaucoup de prudence car les périodes à risques ne sont pas toujours identiques d'une année à l'autre : la quantité de pollens dans l'air peut varier selon la météo (vent, pluie, soleil).

Dans ces conditions le bulletin allergo-pollinique hebdomadaire apparaît comme un outil diagnostique très utile, édité pour la population médecins, malades, dans un but de lutte préventive et curative de l'allergie.

\section{REFERENCES}

1- Asher M.I ., Weiland S.K. The international study of asthma and allergies in childhood (ISAAC). Clin. Exp. Allergy 1998; 28(5): 52-66.

2- Burney $P_{\text {. }}$ Chinn S., Jarvis D., et al. Variations in the prevalence of respiratory symptoms, self-reported asthma attacks, and use of asthma medication in the european community respiratory heathl Survey (ECRH). Eur Respir $\ 1996$; 9(4): 687-695

3- Dhivert H., Donnadieu H., Bousquet J. Pollinose : allergies. Rev Prat 1996; 46 (8): $942-948$

4- Thibaudon M. Cailler J. Evolution temporelle et géographique des principaux pollens allergisants en France : 1987-2000. Allerg Immunol $2001 ; 33(1)$ : 32-36. 5- Charpin J. Atlas européen des pollens allergisants. Rueil-Malmaison : Sandoz; Paris : Edition Groupe Orange, 1974.-p. 125-130.

6-Thibaudon $M$, Cailler J. Evolution temporelle et géographique des principaux pollens allergisants en France de 1987 à 2001 . Allerg Immunol 2002 ; 34(5): 154 -
157.

7- Thibaudon M., Sulmont G. Données aéropolliniques France : année 2001 St.Clément-les-Places : Réseau national de surveillance aérobiologique (R.N.S.A), 2001

8-Mathern G. Ambroisie et étude de sa dissémination. Allerg Immunol 2002 ; 34(4): 129-131.

9- Thibaudon M., Sulmont G. Données aéropolliniques France : année 2002. St.Clément-les-Places : Réseau national de surveillance aérobiologique (R.N.S.A), 2002

10- Quesnel P., Chambaud L., Le Goasterc C., Thibaudon M., Almoussa M. Association entre les concentrations de pollens ef les pollinoses saisonnières : synthèse des études épidémiologiques publiées entre 1978 et 1995. Rev Epidemiol Santé Publique 1997; 45(3) : 224-236. 